\title{
Vernetztes Teammitglied oder isolierte Führungsperson?
}

\section{Netzwerkanalytische Betrachtung von Leitungspersonen in Kindertageseinrichtungen}

\author{
Mareike Bahn, Christoph Weihrather und Hendrik Lohse-Bossenz \\ Pädagogische Hochschule Heidelberg
}

\begin{abstract}
Zusammenfassung: Der Beitrag präsentiert Ergebnisse einer netzwerkanalytischen Betrachtung relationaler Beziehungsstrukturen in Kindertageseinrichtungen (Kitas) bezüglich der Position von Leitungskräften, um Kooperationsstrukturen innerhalb von Teams zu verdeutlichen. Zwei zentrale Aufgabengebiete von Leitungskräften in Kitas wurden dabei betrachtet: die fachliche und organisatorische Gestaltung pädagogischer Arbeit. Die Darstellung der Beziehungsstrukturen in den Kitas erfolgte mittels der Methode der sozialen Netzwerkanalyse (SNA). Die Vernetzung der jeweiligen Teammitglieder sowie die Position der Leitungskräfte konnten mit netzwerkanalytischen Maßzahlen quantifiziert und visualisiert werden. Datengrundlage bildet eine schriftliche Netzwerkbefragung von zehn Kitas. Die Ergebnisse zeigen erstens, dass die Kitaleitungen Hauptansprechpersonen für organisatorische und fachliche Angelegenheiten für ihre Mitarbeitenden sind. Zweitens deuten die Ergebnisse darauf hin, dass Leitungen mit Freistellung häufiger in organisatorischen als fachlichen Fragen konsultiert werden als Leitungskräfte, die nicht vom Gruppendienst freigestellt sind. Die Erkenntnisse geben Anlass dazu, Leitungskonzepte von Kita-Trägern zu diskutieren. Deutlich wurde auch das große Potenzial der SNA für Forschungsvorhaben im frühpädagogischen Bereich.
\end{abstract}

Schlüsselwörter: Kitaleitung, soziale Netzwerkanalyse, Kooperationsstruktur

Integrated Team Member or Isolated Leader? Network Analysis of Leaders in Child Daycare Facilities

Abstract: This article presents the results of a network analysis of the relationships between employees in daycare centers regarding the position of leaders. The goal is to clarify the existing cooperation structures within teams. We consider two central areas of responsibility of managers in daycare facilities, based on their work-specific requirements: the professional and the organizational design of pedagogical work. We used the method of social network analysis to represent the relationship structures. This method quantifies and visualizes the networking of the respective team members and the position of the management staff using network analytic measures. The data are based on a written network survey of ten daycare facilities. The findings indicate that managers of daycare centers are the main contact persons for both the organizational and educational concerns of their employees. The results also suggest that managers who are not active in groupwork are consulted more often on organizational than on pedagogical issues compared to managers who remain active in groupwork. The findings give reason to further discuss the management concepts of daycare facility providers. In addition, this article highlights the great potential of social network analysis for research projects in early childhood education.

Keywords: daycare manager, social network analysis, cooperation structures

\section{Theoretischer Hintergrund}

Leitungskräfte in Kindertageseinrichtungen (Kitas) müssen vielfältige und komplexe Anforderungen bewältigen. Vergegenwärtigt man sich die Führungsspanne, d.h. die einer Führungskraft unterstellten Mitarbeitenden, so wird deutlich, dass Führung maßgeblich über ein aktives Managen von Kooperationen in einem relationalen Netzwerk erfolgt. Der Fokus, Leitungsaufgaben aus solch einer netzwerkana- lytischen Perspektive zu betrachten, findet sich in der frühpädagogischen Forschung bislang wenig (Rehrl \& Gruber, 2007). Der vorliegende Beitrag soll daher die Gestaltung kooperativer Arbeitsbeziehungen als zentrale Leitungsaufgabe in Kitas inhaltlich verorten und mit der Methode der sozialen Netzwerkanalyse (SNA) einen forschungsmethodischen Ansatz präsentieren, wie relationale Beziehungen in Kitas abgebildet und differenzierten Analysen zugänglich gemacht werden können. Auf der Basis einer Untersuchung 
in zehn Kitas mit insgesamt 106 Fachkräften wird der Zusammenhang zwischen einer Freistellung der Kitaleitungen vom Gruppendienst mit der Position innerhalb des kooperativen Netzwerks in der Kita aufgezeigt.

\section{Leitungskräfte und Kooperationen in Kindertageseinrichtungen}

Schwierigkeiten bei der Personalsuche, die Intensivierung der Elternzusammenarbeit, erhöhte pädagogische Herausforderungen sowie stetig veränderte Teamzusammensetzungen mit einer Steigerung der Multiprofessionalität sind nur einige Faktoren, die die Anforderungen an Kitaleitungen in den letzten Jahren erhöht haben. Dabei werden die geringen Zeitressourcen, die den Kitaleitungen für Leitungsaufgaben zur Verfügung stehen, oftmals bemängelt. Zwar kann eine ,zunehmende Anerkennung dieser Tätigkeit und der hiermit verbundenen Steuerungsanforderungen seitens der Kita-Träger" (Autorengruppe Fachkräftebarometer, 2019, S.77) beobachtet werden, dennoch stehen in $10 \%$ der Kitas weiterhin keine expliziten zeitlichen Ressourcen für Leitungsaufgaben zur Verfügung. In den Kitas, in denen die Führungskräfte ein festes Zeitbudget für Leitungstätigkeiten haben, liegt die durchschnittliche Wochenstundenzahl für Leitungsstunden bei 2,1 Stunden (Autorengruppe Fachkräftebarometer, 2019, S.77). Ebert (2009) konstatierte zurecht, dass die Leitungstätigkeiten umso größer sind, je mehr Kinder in der Kita betreut werden und, damit einhergehend, je mehr Fachkräfte in der Einrichtung beschäftigt sind. Die mit dieser Zunahme verbundene Vergrößerung der Führungsspanne (Anzahl direkt unterstellter Personen) und Leitungsintensität wird durch eine (teilweise) Freistellung von der pädagogischen Arbeit im Gruppendienst kompensiert.

Dabei wird Leitungskräften von Kitas eine „Schlüsselposition im Hinblick auf die Integration der pädagogischen und organisationsbezogenen Anforderungen nach innen, die Vertretung der Kindertageseinrichtung nach außen sowie ihre zukunftsorientierte Weiterentwicklung zugewiesen" (Autorengruppe Fachkräftebarometer, 2014, S.35). Die besondere Herausforderung, die mit der Tätigkeit als Leitungskraft einhergeht, ist demnach, eine Symbiose von pädagogischen und organisatorischen Anforderungen herzustellen (Autorengruppe Fachkräftebarometer, 2014). Vor dem Hintergrund des Größenwachstums der Kitas der letzten Jahre von durchschnittlich 7,5 auf elf Mitarbeitende (inklusive Leitungen) und der Nachfrage nach Betreuungsplätzen prognostizierte die Autorengruppe Fachkräftebarometer (2019) eine weitere Steigerung des pädagogischen Kitapersonals. Mit dieser Ausweitung der Führungsspanne gilt es für die Leitungskräfte umso mehr, die beschriebenen Anforderungen insoweit zu steu- ern, dass die pädagogischen Fachkräfte bestmöglich gemeinsam agieren können. Demnach sind Leitungen für das Herstellen von optimalen kooperativen Arbeitsbedingungen sowie für die Personal- und Teamführung zuständig, tragen die pädagogische Verantwortung und überblicken die organisatorischen Abläufe der Kita.

Strehmel (2018) konstatierte diesbezüglich, dass unter anderem die Motivation, die Kompetenz, die Autonomie sowie die Reflexion des pädagogischen Alltags der Mitarbeitenden von der Leitung maßgeblich bestimmt werden. Dass die pädagogische Qualität umso höher ist, je höher sich die Leitungsqualifikation darstellt, belegen Ergebnisse der britischen EPPE-Studie (Effective Preschool and Primary Education) für den Kita-Bereich (Sylva, Melhuish, Sammons, Siraj \& Taggart, 2004). Wertfein, Wildgruber, Wirts und Becker-Stoll (2013) wiederum konnten in ihrer Studie den Zusammenhang zwischen einer positiven Kooperation unter den Fachkräften und der Fachkraft-Kind-Interaktion aufzeigen. Welchen Einfluss die Leitungsqualität auf die Teamqualität und damit auf die pädagogische Prozessqualität hat, wird aktuell im Projekt Leitungsqualität in Kindertageseinrichtungen (LeiKi) untersucht (Heß, Eling, Schmidt \& Smidt, 2021). Zusammenfassend zeigt sich, dass die Bedeutsamkeit der Teamzusammenarbeit auch im Hinblick auf die pädagogische Prozessqualität (Wertfein, Wildgruber, Wirts \& Becker-Stoll, 2013; Lochner, 2017) sowie die Bedeutung und der Einfluss, den die Leitungskraft auf das Team bzw. Organisation und damit einhergehend auf die Prozessqualität der Kita hat (Heß et al., 2021; Viernickel, Nentwig-Gesemann, Nicolai, Schwarz \& Zenker, 2013; Strehmel \& Ulber, 2014), zunehmend in den Fokus der Forschung rücken. Forschungsansätze, die relationale Beziehungsstrukturen und Kooperationsbeziehungen innerhalb von Kita-Teams aus einer netzwerkanalytischen Perspektive untersuchen und dabei die Zusammenarbeit mit den Leitungskräften betrachten, sind aktuell wenig präsent (Rehrl \& Gruber, 2007). Beispielhaft stellen sich dabei Fragen danach, in welcher Form, zu welchen Themen und in welcher Häufigkeit die Kitaleitungen mit den Fachkräften kooperieren bzw. zu welchen Themen sie Ansprechperson für die Fachkräfte sind. Dies kann Aufschluss über die komplexen Zusammenarbeitsbeziehungen geben und bei der Ableitung von Organisations- und Personalentwicklungsmaßnahmen unterstützen.

\section{Soziale Netzwerkanalyse (SNA) als forschungsmethodischer Ansatz}

Soziale Netzwerke stehen im Fokus der SNA. Im Sinne einer Kita besteht ein Netzwerk aus einer nach außen abgegrenzten Menge an Personen, den sogenannten Knoten, die sich durch die Beziehungen und Relationen, den Kan- 
ten, der Individuen untereinander auszeichnet. Die Relationen lassen sich nach Jansen (2006) zufolge hinsichtlich des Inhalts, der Intensität und der Form unterscheiden. Im Rahmen der vorliegenden Studie wurde die Kommunikation, im Sinne eines Austauschs von Informationen, als Relationsinhalt erhoben. Die Relationsintensität wurde anhand von Angaben zur Häufigkeit der Kommunikation zwischen den verschiedenen Individuen erfasst. In Bezug auf die Form der Relation handelt es sich jeweils um gerichtete Beziehungen. Im Rahmen einer Kita kann die Beziehung zwischen zwei Akteuren somit vier Ausprägungen haben: Es besteht keine Beziehung, es besteht eine einseitige Beziehung von Akteur A zu B, es besteht eine einseitige Beziehung von Akteur $\mathrm{B}$ zu A, es besteht eine gegenseitige Beziehung von $\mathrm{A}$ zu B und von B zu A. Zur Auswertung dieser Form relationaler Daten liefert die SNA einen forschungsmethodischen Ansatz, der auch für den frühpädagogischen Kontext als gewinnbringend erachtet werden kann (Rehrl \& Gruber, 2007). Mit Hilfe der SNA können quantifizierte Befunde zur Teamkooperation in Kitas unter Berücksichtigung verschiedener individueller Merkmale der Akteure aufgezeigt werden. Die SNA liefert hierzu Maßzahlen zur Dichte, Gradzentralität und In- bzw. OutDegree. Die Dichte beschreibt das faktische Verhältnis der vorhandenen Beziehungen zu allen grundsätzlich möglichen Beziehungen innerhalb einer Organisation (Jansen, 2006). Diese kann einen Wert zwischen 0 (alle Akteure sind isoliert) und 1 (alle Akteure sind miteinander verbunden) annehmen (Lohse-Bossenz \& Ratzlaff, 2018). Hierdurch erhält man einen Einblick, wie stark bzw. schwach die Fachkräfte in einer Kita miteinander vernetzt sind. Die Größe des Netzwerks beeinflusst die Dichte, so nimmt sie mit der Größe des Netzwerks tendenziell ab. Die Dichte liefert Informationen über den Kooperationsgrad innerhalb einer Kita, wobei eine große Dichte auf einen hohen Kooperationsgrad hinweist. Die Gradzentralität (Degree) erfasst die Anzahl der ein- und ausgehenden Verbindungen eines Individuums. Sie liefert demnach Informationen darüber, wie viele Beziehungen eine Fachkraft in einer Kita im Netzwerk besitzt und folglich, wie hoch der „Grad der Verflechtung“ (Schnegg \& Lang, 2002, S.37) ist. Personen mit einer hohen Gradzentralität sind essenziell für die Verbreitung von Informationen und Wissen, da sie Kontakte zu vielen Personen im Netzwerk besitzen (Müller-Prothmann, Siegberg \& Finke, 2005). Die Gradzentralität oder auch der sogenannte Degree wird in den In-Degree und den Out-Degree differenziert. Während der In-Degree alle empfangenen Beziehungen einer Person (d.h. wie oft wird die Person von anderen angesprochen) beschreibt, spiegelt der Out-Degree die ausgehenden Beziehungen des Individuums (d.h. wie viele Personen spricht eine Person an) wider (Schnegg \& Lang, 2002). Personen mit einem hohen In-Degree bezeichnete
Jansen (1999) als „prominente Akteure“ (S.122), da sie Zugang zu vielen Informationen und Netzwerkressourcen sowie Kontrollmöglichkeiten besitzen.

\section{Fragestellung der Studie}

Das Anliegen dieser Studie bestand darin, kooperative Strukturen von Kita-Teams mit Hilfe der SNA abzubilden. Es wurde untersucht, wie sich Leitungskräfte abhängig davon, ob sie für Leitungsaufgaben freigestellt sind oder nicht, im Netzwerk positionieren. Dabei wurde angenommen, dass Leitungen eine hohe Zentralität und überdurchschnittlich viele eingehende Beziehungen im Netzwerk aufweisen. Zudem wurde erwartet, dass sich Netzwerke vor dem Hintergrund der arbeitsspezifischen Anforderungen der Leitungskräfte aufbauen. Daraus abgeleitet wurde weiterhin angenommen, dass Leitungskräfte, die für Führungsaufgaben freigestellt sind, in ihren Netzwerken bezüglich organisatorischer Anliegen zentral und in fachlichen Fragen weniger zentral positioniert sind. Anhand der Kennzahlen zur Dichte und Gradzentralität wird das Erkenntnispotential der SNA für die Untersuchung von Teamstrukturen generell und der Rolle von Leitungskräften im Speziellen deutlich.

\section{Methode}

\section{Stichprobe und Durchführung}

Die Stichprobe wurde in Zusammenarbeit mit einem Träger von Kitas aus der Rhein-Neckar-Region rekrutiert. Hierdurch konnten einheitliche Grundvoraussetzungen (z.B. hinsichtlich pädagogischem Konzept, übergeordneter Trägerstruktur, finanziellen Ressourcen) gewährleistet werden. Insgesamt nahmen 106 pädagogische Fachkräfte aus zehn verschiedenen Kindertagesstätten an der Untersuchung teil. Die Größe der Einrichtungen lag zwischen fünf und 18 Mitarbeitenden. 13 Personen gaben an, aktiv Leitungstätigkeiten zu übernehmen. Die Strukturen des Trägers sind so geregelt, dass eine Leitungskraft mit mindestens zehn Mitarbeitenden vom Gruppendienst freigestellt ist. Die drei stellvertretenden Leitungskräfte sind mit reduziertem Umfang weiterhin im Gruppendienst tätig. Dies trifft auf die Hälfte der beteiligten Einrichtungen zu. Von den 106 Fachkräften waren 99 weiblich $(93,4 \%)$ und 7 männlich (6,6\%). Unter den pädagogischen Fachkräften befanden sich 11 Akademiker_innen mit einem Abschluss als Sozialpädagog_in oder Kindheitspädagog_in (10,4\%). Die restlichen Fachkräfte hatten eine Ausbildung als Erzieher_in oder Kinderpfleger_in (89,6\%). Die Altersver- 
teilung der Studie entspricht der bundesweiten Altersverteilung der pädagogischen Fachkräfte im Jahr 2018 (Autorengruppe Fachkräftebarometer 2019, S. 33). 28,15\% waren $<30$ Jahre, 20,39\% waren zwischen 30-40 Jahre und 51,46 \% der Fachkräfte waren $>40$ Jahre. Die durchschnittliche Berufserfahrung der pädagogischen Fachkräfte lag bei $M=14.2$ Jahre $(S D=11.4)$. Personen, die am Tag der Erhebung in der jeweiligen Kita nicht anwesend waren, wurden bei der Netzwerkanalyse nicht einbezogen.

\section{Instrument}

Die Positionierung der Leitungskräfte im Netzwerk in Abhängigkeit einer Leitungsfreistellung wurde mit Hilfe eines eigens entwickelten Fragebogens erhoben. In Anlehnung an Ratzlaff und Lohse-Bossenz (2018) sowie auf Grundlage der bereits beschriebenen arbeitsspezifischen Anforderungen von Leitungskräften in Kitas erfolgte die Betrachtung hinsichtlich der zwei zentralen Aufgabengebiete: der fachlichen sowie der organisatorischen Gestaltung pädagogischer Arbeit in Kitas (Relationsinhalt). Es wurde gefragt, ob eine Person jeweils „Ansprechpartner in für fachliche und/oder Ansprechpartner_in für organisatorische Angelegenheiten“ ist. Zudem wurde die Häufigkeit des Kontakts jedes Teammitglieds der Kita mit jedem anderen potenziellen Mitglied erfasst (Relationsintensität). Die Skala zur Kontakthäufigkeit reichte dabei von „selten“ über „manchmal“ bis „oft“. Die Teilnehmenden gaben weiterhin über ein dichotomes Antwortformat (,Ja/Nein") an, mit welchen Personen sie gemeinsam in einer Gruppe arbeiten. Somit gab jedes Teammitglied für jedes andere Mitglied an, ob sie zusammen in einer Gruppe arbeiten und ob die jeweilige Person angesprochen wird (Relationsform). Auf diese Weise können auch bidirektionale Verbindungen identifiziert werden.

Ein Vorteil der SNA im Gegensatz zu anderen Untersuchungen hinsichtlich der Kooperation in Kitas ist, dass die Erhebung über eine reine Selbsteinschätzung einzelner Personen, wie beispielsweise der Leitung, hinausgeht, da von allen Fachkräften innerhalb einer Kita Daten vorliegen. In einer kleinen Kita mit fünf Fachkräften resultieren somit 60 individuelle Einschätzungen, in einer großen Kita mit 21 Fachkräften dementsprechend 1260.

\section{Analytisches Vorgehen}

Die Netzwerkanalyse zur Identifizierung der Positionierung der Leitungskräfte erfolgte anhand des Excel-Tools NodeXL (Smith, Ceni, Milic-Frayling, Shneiderman, Mendes Rodrigues, Leskovec \& Dunne, 2010). Zur Beantwortung der Frage, wie Leitungskräfte in Teams in Abhängig- keit davon, ob sie für die Leitungsaufgaben freigestellt sind, positioniert sind, wurde die Gradzentralität auf individueller Ebene als zentraler Kennwert herangezogen. Für jede der zehn Einrichtungen wurden jeweils zwei Netzwerke erstellt - eines für den organisatorischen und eines für den fachlichen Austausch. Aus den Netzwerken lassen sich sowohl Kennwerte auf der Ebene der Netzwerke als auch auf der Ebene individueller Personen ableiten. Ein Vergleich auf Ebene des Gesamtnetzwerks kann als problematisch angesehen werden, da die Freistellung für Leitungsaufgaben mit der Einrichtungsgröße konfundiert ist. Dies trifft auch für Individualparameter zu, jedoch erfolgte hier ein ipsativer Vergleich zwischen organisatorischen und fachlichen Netzwerken sowie von unterschiedlichen Personengruppen innerhalb des Teams und der Freistellung. Die Gradzentralität mit den Facetten In- und OutDegree stellte ein Maß für die direkte Kontaktaufnahme hinsichtlich fachlicher und organisatorischer Fragen dar und erscheint daher sinnvoll, herangezogen zu werden, um die Annahme zu prüfen, dass die Positionierung von Leitungskräften abhängig von einer Leitungsfreistellung ist. Für den Vergleich zwischen Leitungskräften und zugeordneten pädagogischen Fachkräften wurden die Gradzentralitätsparameter der Leitungskräfte mit der Gradzentralität der pädagogischen Fachkräfte verglichen und auf Signifikanz geprüft. In einem zweiten Schritt erfolgte eine varianzanalytische Prüfung, bei dem Gradzentralitätsparameter für den fachlichen und organisatorischen Austausch als Within-subjects-Faktoren modelliert wurden. Position im Team (Pädagogische Fachkraft vs. Leitungskraft) und Leitungsfreistellung (ohne Freistellung vs. mit Freistellung) wurden als Between-subjects-Faktoren einbezogen.

\section{Ergebnisse}

Tabelle 1 zeigt die Ergebnisse der organisatorischen und fachlichen Zusammenarbeit anhand der individuellen Gradzentralität sowie dem In- und Out-Degree unter Berücksichtigung der Position im Team. Es wird deutlich, dass die Leitungskräfte, wie angenommen, überdurchschnittlich viele Beziehungen im Netzwerk haben. Der überdurchschnittliche In-Degree ist Beleg dafür, dass die Leitungen für viele Mitarbeitende als fachliche und organisatorische Ansprechpartner_innen gelten. Beim OutDegree zeigten sich weder in fachlicher noch organisatorischer Hinsicht signifikante Unterschiede. Interessant ist in diesem Zusammenhang der Unterschied zwischen Inund Out-Degree hinsichtlich organisatorischer Anliegen. Es zeigte sich, dass Leitungskräfte besonders häufig Ansprechperson für organisatorische Anliegen sind, ohne selbst eine entsprechende Ansprechperson zu haben. 
Tabelle 1. Netzwerkparameter für Leitungskräfte in Abhängigkeit von der Position im Einrichtungsteam

\begin{tabular}{|c|c|c|c|c|c|c|c|c|}
\hline & Position* & $N$ & $M$ & $S D$ & $t$ & $d f$ & $p$ & $d$ \\
\hline \multicolumn{9}{|c|}{ Fachlicher Austausch } \\
\hline \multirow[t]{2}{*}{ Degree } & 1 & 92 & 4.65 & 2.10 & -2.23 & 14.4 & 0.04 & -0.71 \\
\hline & 2 & 13 & 6.31 & 2.56 & & & & \\
\hline \multirow[t]{2}{*}{ In-Degree } & 1 & 92 & 3.08 & 2.13 & -3.17 & 13.9 & 0.01 & -1.04 \\
\hline & 2 & 13 & 5.69 & 2.87 & & & & \\
\hline \multirow[t]{2}{*}{ Out-Degree } & 1 & 92 & 3.47 & 1.83 & 0.85 & 14.4 & 0.41 & 0.27 \\
\hline & 2 & 13 & 2.92 & 2.22 & & & & \\
\hline \multicolumn{9}{|c|}{ Organisatorischer Austausch } \\
\hline \multirow[t]{2}{*}{ Degree } & 1 & 92 & 4.61 & 1.82 & -3.44 & 13.0 & $<.01$ & -1.22 \\
\hline & 2 & 13 & 7.92 & 3.40 & & & & \\
\hline \multirow[t]{2}{*}{ In-Degree } & 1 & 92 & 2.92 & 1.80 & -4.74 & 13.0 & $<.01$ & -1.68 \\
\hline & 2 & 13 & 7.46 & 3.38 & & & & \\
\hline \multirow[t]{2}{*}{ Out-Degree } & 1 & 92 & 3.51 & 1.70 & 0.36 & 14.7 & 0.72 & 0.11 \\
\hline & 2 & 13 & 3.31 & 1.93 & & & & \\
\hline
\end{tabular}

Anmerkungen: * 1 = pädagogische Fachkraft, 2 = Fachkraft mit Leitungsaufgaben; $M=$ Mittelwert, $S D=$ Standardabweichung, SE = Standardfehler, $t=$ Test größe aus Welch-Test für unabhängige Stichproben, $d f=$ Freiheitsgrade, $p=$ Irrtumswahrscheinlichkeit, $d$ = Cohens $d$ als Effektstärke.

In einem weiteren Schritt wurde nun geprüft, wie sich die verschiedenen Zentralitätsparameter in Abhängigkeit von der Position in der Einrichtung und Freistellung für Leitungsaufgaben verändern. Tabelle 2 zeigt die Ergebnisse der Varianzanalysen, wobei drei separate Analysen mit jeweils einem spezifischen Zentralitätsparameter als abhängige Variable gerechnet wurden. Bei der ersten Analyse zur Gradzentralität ist erkennbar, dass der Haupteffekt des betrachteten Relationsinhalts (fachlich vs. organisatorisch) signifikant ist $(F(1)=4.48, \mathrm{p}<.05)$. Auch die Interaktionen zwischen den betrachteten Variablen sind signifikant. Aus Abbildung 1 zur graphischen Interpretation ist erkennbar, dass in Einrichtungen ohne Leitungsfreistellung die Leitungskräfte sowohl fachlich als auch organisatorisch ähnlich bedeutsame Ansprechpersonen wie ande- re pädagogische Fachkräfte sind (der visuell erkennbare Unterschied ist statistisch nicht signifikant). In Einrichtungen mit Leitungsfreistellung findet sich diese Gleichverteilung nicht - hier ist der Unterschied hinsichtlich der organisatorischen Aspekte höher als beim fachlichen Austausch.

\section{Diskussion}

Leitung und Führung in Kitas als aktives Management von Interaktionsstrukturen zu begreifen, bildete den Ausgangspunkt der vorliegenden Studie. Vor diesem Hintergrund wurde die SNA als eine Methode der direkten Erfassung
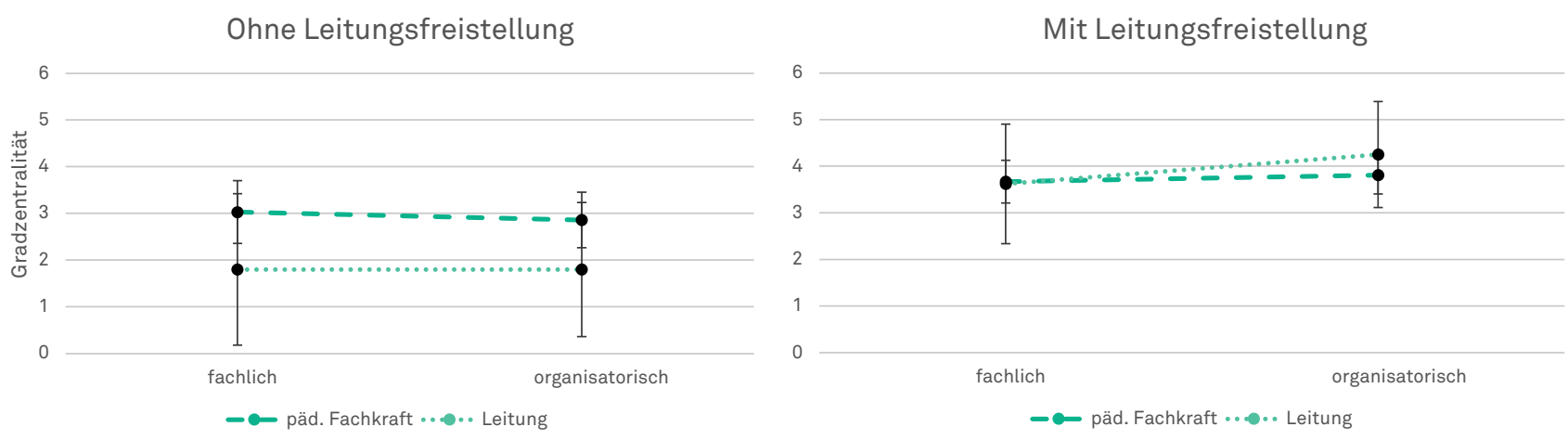

Abbildung 1. Graphische Darstellung der Interaktion fachlich/organisatorisch * ohne Leitungsfreistellung/mit Leitungsfreistellung (o.LFS/m. LFS) * Position in der Einrichtung. 
Tabelle 2. Ergebnisse der Varianzanalysen mit Messwiederholung/verbundene Stichproben für Relationsinhalt (fachlich vs. organisatorisch) und den Faktoren Position (pädagogische Fachkraft vs. Fachkraft mit Leitungsaufgaben) und Leitungsfreistellung (mit vs. ohne) sowie der Interaktion der Faktoren (*)

\begin{tabular}{|c|c|c|c|c|}
\hline & $F$ & $d f$ & $p$ & $\eta_{p}^{2}$ \\
\hline \multicolumn{5}{|l|}{ Degree } \\
\hline Relationsinhalt & 4.48 & 1 & 0.04 & 0.04 \\
\hline Relationsinhalt * Position & 5.85 & 1 & 0.02 & 0.06 \\
\hline Relationsinhalt * Freistellung & 6.12 & 1 & 0.02 & 0.06 \\
\hline Relationsinhalt * Position * Freistellung & 4.25 & 1 & 0.04 & 0.04 \\
\hline \multicolumn{5}{|l|}{ In-Degree } \\
\hline Relationsinhalt & 5.30 & 1 & 0.02 & 0.05 \\
\hline Relationsinhalt * Position & 8.57 & 1 & 0.00 & 0.08 \\
\hline Relationsinhalt * Freistellung & 3.08 & 1 & 0.08 & 0.03 \\
\hline Relationsinhalt * Position * Freistellung & 2.10 & 1 & 0.15 & 0.02 \\
\hline \multicolumn{5}{|l|}{ Out-Degree } \\
\hline Relationsinhalt & 0.36 & 1 & 0.55 & 0.00 \\
\hline Relationsinhalt * Position & 0.44 & 1 & 0.51 & 0.00 \\
\hline Relationsinhalt * Freistellung & 0.90 & 1 & 0.35 & 0.01 \\
\hline Relationsinhalt * Position * Freistellung & 0.10 & 1 & 0.76 & 0.00 \\
\hline
\end{tabular}

Anmerkungen: $F=$ Prüfgröße, $d f=$ Freiheitsgrade, $p=$ Irrtumswahrscheinlichkeit, $\eta_{p}^{2}=$ partielles Eta-Quadrat als Effektstärke.

relationaler Strukturen herangezogen und untersucht, wie sich die Positionierung der Leitungskräfte zwischen Einrichtungen unterscheiden - abhängig davon, ob die Leitungskraft für die Leitungsaufgaben freigestellt ist oder zusätzlich weitere Aufgaben in der Kita übernimmt. Die Leitungskraft, als prominent handelnde Person im Sinne der Netzwerkforschung, besitzt eine zentrale Position bezüglich der Informationsverbreitung im Netzwerk. Die Ergebnisse bestätigten die Annahme, dass Leitungskräfte maßgeblich als organisatorische und fachliche Ansprechperson gesehen werden. Sie weisen eine hohe Zentralität und überdurchschnittlich viele eingehende Beziehungen im Netzwerk auf. Es wird deutlich, dass sich die Arbeitsschwerpunkte wie angenommen von freigestellten und nicht freigestellten Leitungskräften dabei unterscheiden: Eine Leitungskraft mit verbundener Gruppentätigkeit wird von den Mitarbeitenden häufiger in fachlichen sowie organisatorischen Fragen konsultiert. Sind Leitungskräfte vom Gruppendienst freigestellt, werden diese durch die größere Anzahl an Mitarbeitenden zwar generell häufiger als Ansprechperson genannt, jedoch weniger stark als fachliche Ansprechperson, sondern eher als zentrale Ansprechperson für organisatorische Fragen. Dieser Befund deckt sich mit den Erwartungen, da Leitungskräfte letztlich vom aktiven Gruppendienst freigestellt sind, um sich den mit großen Einrichtungen verbundenen umfangreicheren organisatorischen Leitungsaufgaben zu widmen. Aus den Befunden lässt sich an dieser Stelle vorsichtig schließen, dass Leitungskräfte in größeren Einrichtungen eher als or- ganisatorische Ansprechpersonen gesehen werden. Warum Leitungskräfte, die vom Gruppendienst freigestellt sind, häufiger für organisatorische Anliegen und weniger für fachliche Anliegen kontaktiert werden, kann an dieser Stelle nur vorsichtig interpretiert werden. Es lassen sich zwar unterschiedliche Erklärungsansätze formulieren, zu deren Verifizierung weitere Analysen notwendig sind. Zum einen kann dies durch das Leitungshandeln der Führungskraft determiniert werden. Nentwig-Gesemann, Nicolai und Köhler (2016) haben verschiedene Leitungstypen identifiziert. Der fürsorgliche Leitungstypus, der das persönliche Gespräch pflegt, kann dabei eher als fachliche und organisatorische Ansprechperson gelten als der Leitungstyp Management, der eine klare Trennung zieht zwischen den „Leitungsaufgaben und dem pädagogischen Kerngeschäft" (Nentwig-Gesemann et al. 2016, S.38). Gleichzeitig kann auch eine wahrgenommene Distanz durch die Freistellung von Seiten der Fachkräfte ein möglicher Erklärungsansatz sein. Durch die klare Trennung zwischen Leitungsfunktion und Gruppendienst entfallen Türund-Angel-Gespräche mit der Leitung, weshalb für pädagogische Anliegen eher die Kolleg_innen zu Rate gezogen werden. Zudem kann bedingt durch die Größe der Einrichtung über gruppenbasierte Konzepte de facto eine zweite Managementebene mit den jeweils Gruppenverantwortlichen eingeführt worden sein. Die fachlichen Aufgaben werden dadurch in gewissem Maße dezentralisiert, wohingegen die organisatorischen Anliegen weiterhin durch die Leitung und gegebenenfalls spezielle verant- 
wortliche Personen einrichtungsweit bearbeitet werden. Damit verschieben sich die Arbeitsinhalte der Leitungen maßgeblich in Richtung organisatorischer Themen. Weiterführend stellt sich dann die Frage, wie Führungskräfte die eigene Rolle definieren möchten. Vor allem im Falle dessen, dass Fachkräfte aus dem Gruppendienst in eine Leitungsposition wechseln, sollten flankierende Personalentwicklungsmaßnahmen darauf abzielen, entsprechende realistische Erwartung an das neue Aufgabenfeld zu generieren.

\section{Potenzial der sozialen Netzwerkanalyse zur Darstellung kooperativer Arbeitsbeziehungen in Kitas}

Die SNA als Methode bietet über die hier berichteten Analysen hinaus vielfältige Möglichkeiten (Rehrl \& Gruber, 2007), um beispielsweise Substrukturen und Informationsflüsse in einer Einrichtung abzubilden. Gruppenbasierte Organisationsstrukturen sollten sich entsprechend in einer Netzwerkstruktur mit mehreren kleineren Netzwerken zeigen. Außerdem können zentrale Personen für die Weitergabe von Informationen identifiziert werden, denen im Sinne netzwerkorientierter Führung besondere Bedeutung zukommt. Damit kommt der SNA nicht nur im Rahmen der Forschung zu Teamstrukturen und Führungshandeln eine hohe Bedeutsamkeit zu, sondern kann auch spezifisch in den Einrichtungen genutzt werden, um Teamstrukturen zu visualisieren und aktiv zu gestalten.

\section{Einschränkungen der Studie}

Ziel der vorliegenden Studie war es, die SNA als forschungsmethodischen Ansatz zur Untersuchung der Bedeutung von Leitungskräften in Kitas vorzustellen und anhand einer ersten Datenerhebung mögliche Aussagen zu verdeutlichen. Die Stichprobe bestand aus zehn Kitas eines Trägers innerhalb eines Bundeslandes. Daher waren Rahmenbedingungen wie Leitungskonzepte, Trägervorgaben und die föderalen Strukturen des Landes bei den Einrichtungen identisch und die Freistellung der Leitungen konnte spezifisch analysiert werden. Zur Replikation und Erweiterung der vorgestellten, an einer kleinen Stichprobe gewonnen Ergebnisse mit geringen Effektgrößen sind breiter angelegte Studien nötig, um strukturelle Einflussgrößen auf die Zusammenarbeitsbeziehungen herauszuarbeiten. Innerhalb der Untersuchung war es außerdem nur möglich, eine Momentaufnahme bezüglich der Beziehungsstrukturen abzubilden. Dies beinhaltet z. B. die Einschränkung, dass nur die Fachkräfte der Kita an der Studie teilnahmen, die innerhalb des Befragungszeit- raums anwesend waren. Außerdem sind zusätzliche Analysen über die Zeit notwendig, um Aussagen über die Entwicklung von Beziehungs-und Kommunikationsstrukturen innerhalb der Kita treffen zu können. Darüber hinaus wurden Parameter wie z.B. die Betriebszugehörigkeit der Leitungskraft in der Kita oder eine eventuelle Teilzeittätigkeit nicht erfragt bzw. ausgewertet. Aufgrund der Limitierungen ist diese Untersuchung als Pilotstudie zu betrachten, die jedoch das Potenzial der SNA für den frühpädagogischen Kontext verdeutlicht, um zukünftig Beziehungs-, Kommunikations- und Teamstrukturen im Bildungsort Kita zu untersuchen und zu gestalten.

\section{Implikationen für die Personalentwicklung von Leitungskräften}

Die durchgeführte SNA bietet sich auf Praxisebene zur Formulierung von Personalentwicklungsmaßnahmen an. Die Ergebnisse lassen - wie oben schon angeführt - die Vermutung $\mathrm{zu}$, dass die Übernahme von Leitungsaufgaben mit Leitungsfreistellung mit einer Rollenveränderung beim Übergang in eine Leitungsposition einhergehen. Im Sinne der Transparenz und der Sicherung realistischer Erwartungen sollten angehende Leitungskräfte mit dieser Rollenveränderung konfrontiert werden. Hier zeigten diverse Forschungsbefunde, dass realistische Erwartungen an eine Arbeitsstelle in Zusammenhang mit Arbeitszufriedenheit, Arbeitsleistung und Wohlbefinden stehen (Ashforth \& Saks, 2000).

Für Kita-Träger stellt sich die Frage, ob im Zuge von immer größeren betrieblichen Aufgaben durch die steigenden Einrichtungsgrößen neue Leitungskonzepte erarbeitet werden müssen. Eine personelle Differenzierung zwischen „pädagogischer" und „betrieblicher" Leitung, Stellenschaffungen von Verwaltungskräften oder die bewusste Stärkung von Subteamstrukturen, die die betrieblichen Abläufe der Kita in ähnlicher Weise wie die Leitungskraft überblicken, sind hier beispielhaft zu nennen. In Einrichtungen, in denen die fachlichen und organisatorischen Aufgaben in einer Stelle gebündelt sind, wird empfohlen, die Leitungskräfte in diesen Tätigkeiten ausreichend zu unterstützen. Es sollten genügend Zeitkontingente für fachliche und betriebliche Aufgaben bereitgestellt und Weiterbildungen für Management- und Führungskompetenzen gefördert werden.

\section{Literatur}

Ashforth, B. E. \& Saks, A. M. (2000). Personal control in organizations: A longitudinal investigation with newcomers. Human Re- 
lations, 53(3), 311 -339. https://doi.org/10.1177/0018726700 533002

Autorengruppe Fachkräftebarometer (2014). Fachkräftebarometer Frühe Bildung 2014. Weiterbildungsinitiative Frühpädagogische Fachkräfte. München: Deutsches Jugendinstitut e.V.

Autorengruppe Fachkräftebarometer (2019). Fachkräftebarometer Frühe Bildung 2019. Weiterbildungsinitiative Frühpädagogische Fachkräfte. München: Deutsches Jugendinstitut e.V.

Ebert, S. (2009). Was Führen und Leiten in Kitas so schwer macht. Erklärung einer widersprüchlichen Situation und was Leitung tun muss. kindergarten heute - Das Leitungsheft, 3, 4-8.

Heß, I., Eling, V., Schmidt, T. \& Smidt, W. (2021). Untersuchung der Leitungsqualität in Kindertageseinrichtungen - Das Forschungsprojekt Leiki. Frühe Bildung, 9(1), 56 - 58.

Jansen, D. (1999). Einführung in die Netzwerkanalyse. Opladen: Leske + Budrich.

Jansen, D. (2006). Einführung in die Netzwerkanalyse. (3. überarbeitete Auflage). Wiesbaden: VS Verlag für Sozialwissenschaften / GWV Fachverlage GmbH.

Lochner, B. (2017). Teamarbeit in Kindertageseinrichtungen. Kassel: Springer VS.

Lohse-Bossenz, H. \& Ratzlaff, O. (2018). Making networks visible Möglichkeiten und Grenzen der sozialen Netzwerkanalyse im Kontext von Projektevaluationen. Qualität in der Wissenschaft, 12(4), $93-99$.

Müller-Prothmann, T., Siegberg, A. \& Finke, I. (2005). Leveraging Boundary-spanning Knowledge Community Building. Interventions from a Social Network Analysis in Interorganizational R\&D Environments. In N. Gronau, T. Pawlowsky, T. Schildhauer \& P. Schütt (Hrsg.), Wissensmanagment: Motivation, Organisation, Integration (S. 247 - 254). Poing: CMP-WEKA.

Nentwig-Gesemann, I., Nicolai, K. \& Köhler, L. (2016). KiTa-Leitung als Schlüsselposition. Erfahrungen und Orientierungen von Leitungskräften in Kindertageseinrichtungen. Gütersloh: Bertelsmann Stiftung.

Ratzlaff, O. \& Lohse-Bossenz, H. (2018). Mehr als die Summe seiner Teile - Soziale Netzwerkanalyse in der Projektevaluation von PSI-Potsdam. In A. Borowski, A. Ehlert \& H. Prechtl (Hrsg.), PSI-Potsdam - Ergebnisbericht zu den Aktivitäten im Rahmen der Qualitätsoffensive Lehrerbildung (2015 - 2018) (S.321 - 344). Potsdam: Universitätsverlag.

Rehrl, M. \& Gruber, H. (2007). Netzwerkanalysen in der Pädagogik. Ein Überblick über Methode und Anwendung. Zeitschrift für Pädagogik, 53(2), $243-264$.
Schnegg, M. \& Lang, H. (2002). Netzwerkanalyse. Eine praxisorientierte Einführung. Methoden der Ethnographie, 1, 1- 55.

Strehmel, P. (2018). Kita Leitungen als zentrale Akteure im KitaSystem. Vortrag anlässlich der Fachtagung: Kita-Leitung als Schlüsselposition im System frühkindlicher Bildung, Erziehung und Betreuung, Basthorst.

Strehmel, P. \& Ulber, D. (2014). Leitung von Kindertageseinrichtungen. Weiterbildungsinitiative Frühpädagogische Fachkräfte, WiFF Expertisen, 39. München: Deutsches Jugendinstitut e.V.

Smith, M., Ceni, A., Milic-Frayling, N., Shneiderman, B., Mendes Rodrigues, E., Leskovec, J. \& Dunne, C. (2010). NodeXL: a free and open network overview, discovery and exploration add-in for Excel 2007/2010/2013/2016 from the Social Media Research Foundation. Verfügbar unter: https://www.smrfoundation.org

Sylva, K., Melhuish, E., Sammons, P., Siraj, I. \& Taggart, B. (2004). The Effective Provision of Pre-School Education (EPPE) Project: Findings from Pre-school to end of Key Stage1. Verfügbar unter: https://dera.ioe.ac.uk/18189/2/SSU-SF-2004-01.pdf

Viernickel, S., Nentwig-Gesemann, I., Nicolai, K., Schwarz, S., \& Zenker, L. (2013). Schlüssel zu guter Bildung, Erziehung und Betreuung. Bildungsaufgaben, Zeitkontingente und strukturelle Rahmenbedingungen in Kindertageseinrichtungen. Berlin: Alice-Salomon-Hochschule.

Wertfein, M., Wildgruber, A., Wirts, C. \& Becker-Stoll, F. (2013). Interaktionen in Kindertageseinrichtungen. Göttingen: Vandenhoeck \& Ruprecht.

\section{Autorenschaften}

Die Mitautor_innen sind erreichbar unter: Christoph Weihrather, chrisweihrather@gmail.com; Hendrik Lohse-Bossenz, hendrik. lohse-bossenz@ph-heidelberg.de

\section{Förderung}

Open Access-Veröffentlichung ermöglicht durch die Pädagogische Hochschule Heidelberg.

\section{Mareike Bahn}

Pädagogische Hochschule Heidelberg

Keplerstraße 87

69120 Heidelberg

bahn@ph-heidelberg.de 